\title{
Development of Portable Terminal for Facility Inspection
}

\section{Makoto KIKUCHI}

Senior Researcher,

Laboratory Head,

Facilities Management Systems, Transport Information Technology Division

\section{Michiko NOZUE}

Senior Researcher,

We studied a method of using a portable terminal to input inspection data into a facility management database and undertook research into a prototype data entry system that uses a cellular phone and a Personal Digital Assistant (PDA), applying speech recognition and a method to automatically identify facilities with radio frequency identification (RF-ID) tags. In addition, we conducted research into methods that would enable inspection data entry even in areas where electric wave is interrupted and an e-mail question answering system that facilitates manual information retrieval and simple fault diagnosis is under development. This paper describes the research results concerning the use of the portable terminal.

Keywords: radio frequency identification (RF-ID) tag, facility management, portable terminal, maintenance manual.

\section{Introduction}

Portable terminals have been used for some time in facility maintenance work with the aim of improving efficiency. There were however some problems encountered, such as the size of the operation screen, the weight of the terminal itself and inadequate performance, and it was not in frequent use ${ }^{1)}$. Therefore, we incorporated on an experimental basis and evaluated the following four functions with the aim of using the portable terminal more efficiently in the maintenance field.

- Wireless connection with database server using mobile Internet function

- Automatic identification of facility for inspections using RF-ID tags

- Inspection data input by speech recognition to achieve hands-free operation

- Measures to cope with electric wave interruption as encountered in tunnels

On the other hand, because of the rising demand for instant retrieval of necessary information from huge amounts of stored text data, accompanied with recent improvements in computer performance, capacity and networking, there has been a lot of advanced research conducted into information retrieval and Japanese natural language processing and development of systems that integrate both technologies. Then, as there has been an increasing trend in information retrieval research for systems to be of the question answering type, we have developed a question answering system in the field of facility maintenance.

\section{Facility maintenance characteristics}

On railways, facility maintenance and its work en- vironment have the following characteristics.

a) Electric wave interrupted area

Cellular phones are not usable in tunnels, in areas along railway lines or beside power lines in mountainous districts where base stations are not established. Therefore, it is necessary to think about measures to cope with electric wave interruption or to consider alternative means of communication.

b) Dangers

When undertaking trackside work, it is necessary to confirm train operations have ceased to prevent train contact accident and to ensure that the power is off to prevent high voltage contact accident.

c) Nighttime work

Although it is possible to carry out work for short periods during train interval in the daytime, work that is more time-consuming has to be carried out at night after the last train and before services resume the next day. Lighting equipment such as back-lights or flashlights then becomes indispensable.

d) Temperature

Since the temperatures in which maintenance work is carried out can range from at least -10 degrees $\mathrm{C}$ in winter to over 40 degrees $\mathrm{C}$ in summer, any portable terminal would need to be able to tolerate such wide-ranging temperatures.

e) Dirt

In facility maintenance work, dirt from oil and dust is unavoidable and workers have to operate equipment with hands or gloves covered with oil, so any portable terminal should be adapted to cope with these conditions. f) Input content

With regard to input content, it will be necessary to deal with measurement data of facility inspection, graphical representations for the purpose of confirmation, photographic data showing defects, etc. 


\section{Experimental system}

\subsection{Outline of experimental system}

The system made for trial purposes this time has the following features in connection with the above-mentioned facility maintenance work characteristics.

- Using the PDA's mobile Internet function makes it unnecessary to download data in advance. In addition, by accessing the Web page directly from the work field, we can refer to data, input inspection results and confirm or change work plans.

- As input data is stored immediately onto the database server via the Web page, a work manager in an office can understand the situation in the field in real time.

- Automatic facility identification for inspections is made possible by reading the RF-ID tag without having to retrieve each facility through the menu every time. Therefore, it can improve the efficiency of facility inspection work.

- In areas where electric wave is interrupted such as in tunnels, data is saved in the PDA's memory and when communication becomes possible, the saved data is transmitted to the server automatically. As a result, the PDA data input operation can always be achieved regardless of electric wave reception.

- It is possible to input inspection data using speech recognition.

\subsection{Wireless connection with database}

In this system, data necessary for the inspection such as the inspection plan, the inspection history, and the inspection master is prepared beforehand in the office and downloaded from the server via the Internet. The worker inputs the inspection result while referring to this data at the field. The input data is uploaded to the server in the office via the Internet, and recorded directly onto the database. Figure 1 shows the system structure.

\subsection{Automatic facility identification}

The tag reader connected to a PDA can read data of RF-ID tag attached to the facility to be inspected. On the RF-ID tag, the equipment ID needed to identify the facility is stored beforehand. The facility name and the date of the last inspection are displayed on the screen when retrieving master and inspection information using the equipment ID read by the tag reader. The in-

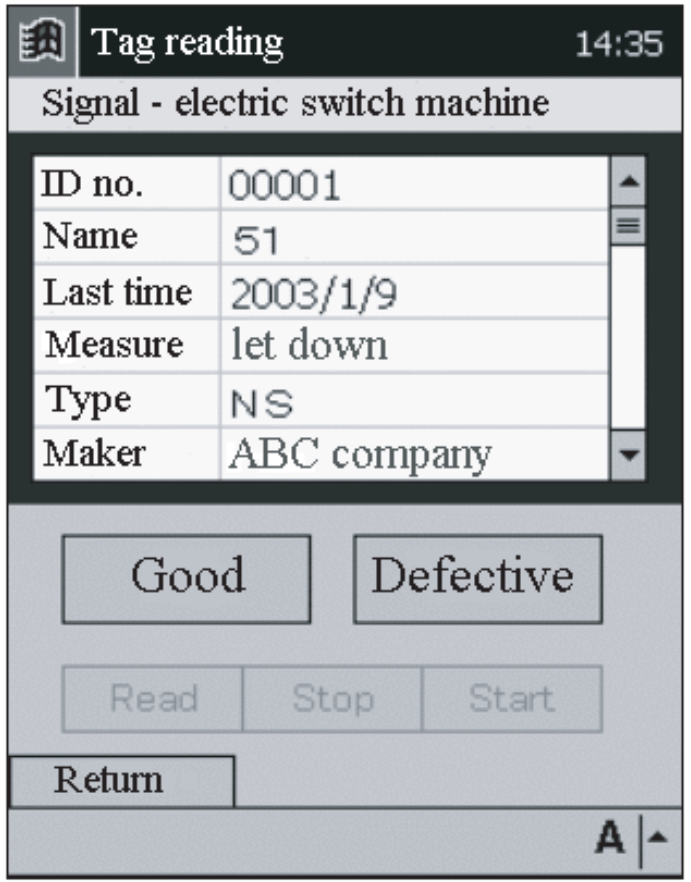

Fig. 2 Tag reading screen

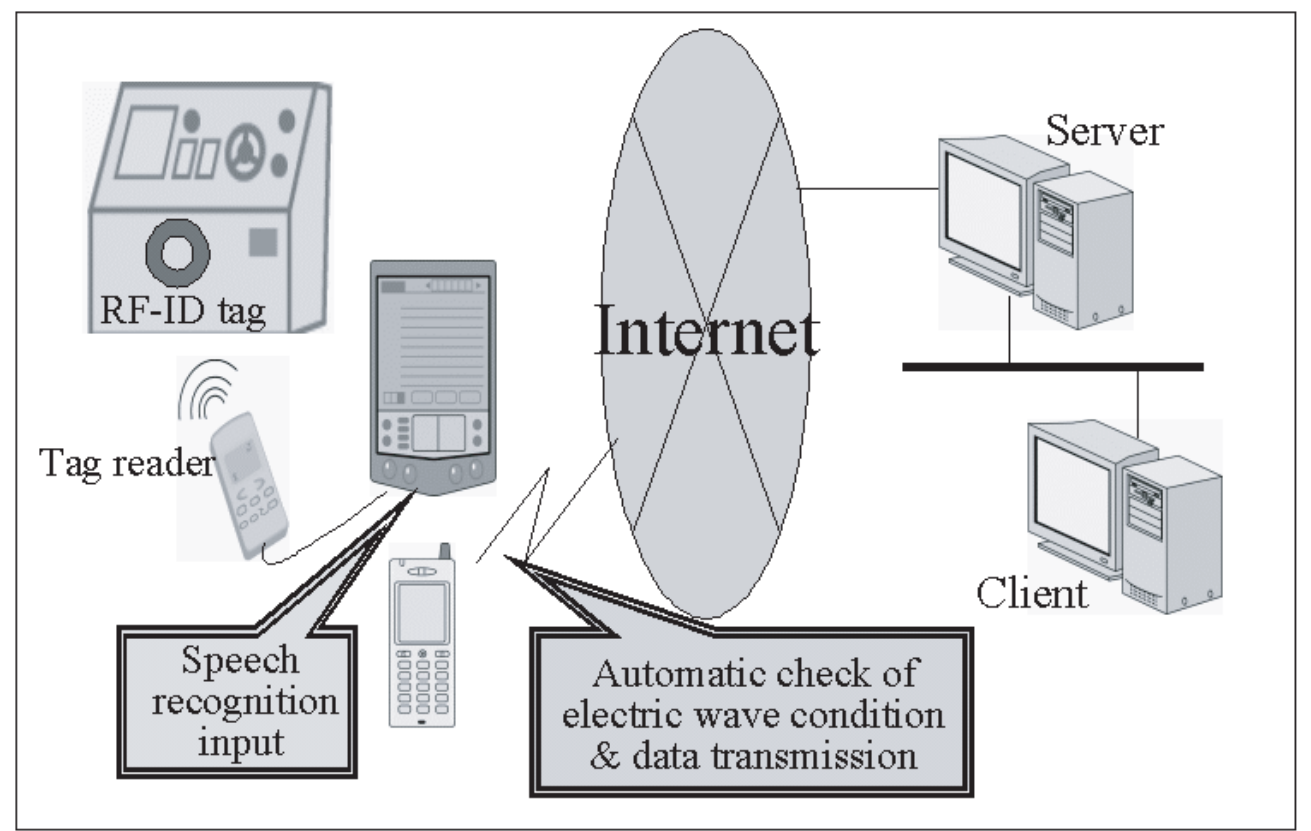

Fig. 1 System structure 
spection result is recorded directly onto the database server in the office by wireless communication such as by cellular phone via the Internet. Figure 2 shows a tag reading screen.

\subsection{Data input using speech recognition}

It is complicated to input inspection data from a small PDA screen and workers have to do two jobs (i.e. inspection and data input) simultaneously. Therefore, for trial purposes, we have developed an input function that uses speech recognition that makes hands-free operation possible when workers input inspection data.

In general, there are two types of speech recognition: word recognition and continuously speech recognition. Because the vocabulary and the items to be input during the inspection are almost fixed, we have adopted a speech recognition method that limits the input pattern, corresponding to a compromise of both methods.

In speech recognition by input pattern, when the inspection result shows the facility conditions to be excellent, we input a voice message such as "(Facility name) Good." On the other hand, if the inspection result shows the facility conditions to be defective, we input the fixed pattern of "Facility name," "Inspected item," "Defective part name," "Type of problem," and "Measure" by voice (Fig. 3). Moreover, if the inspection result shows a facility defect, it is possible to input each pattern in any order because there are a lot of input items.

\subsection{Electric wave interruption}

Cellular phones are not usable in tunnels, in areas along railway lines or beside power lines in mountainous districts where base stations are not established.

\section{Voice input} $+412: 00 \times$

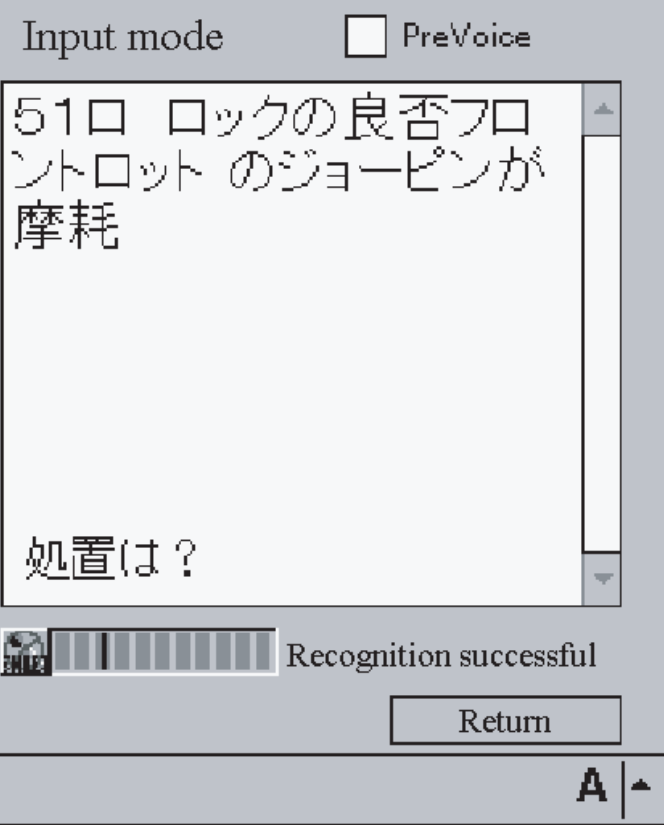

Fig. 3 Speech recognition screen

Therefore, it is necessary to take measures to cope with electric wave interruption in order to use the mobile Internet function. So, we have developed a function to execute the following process.

- Wireless card electric wave status (open or interrupted) is checked by the PDA background process.

- When the electric wave is open, data is recorded immediately onto the database server.

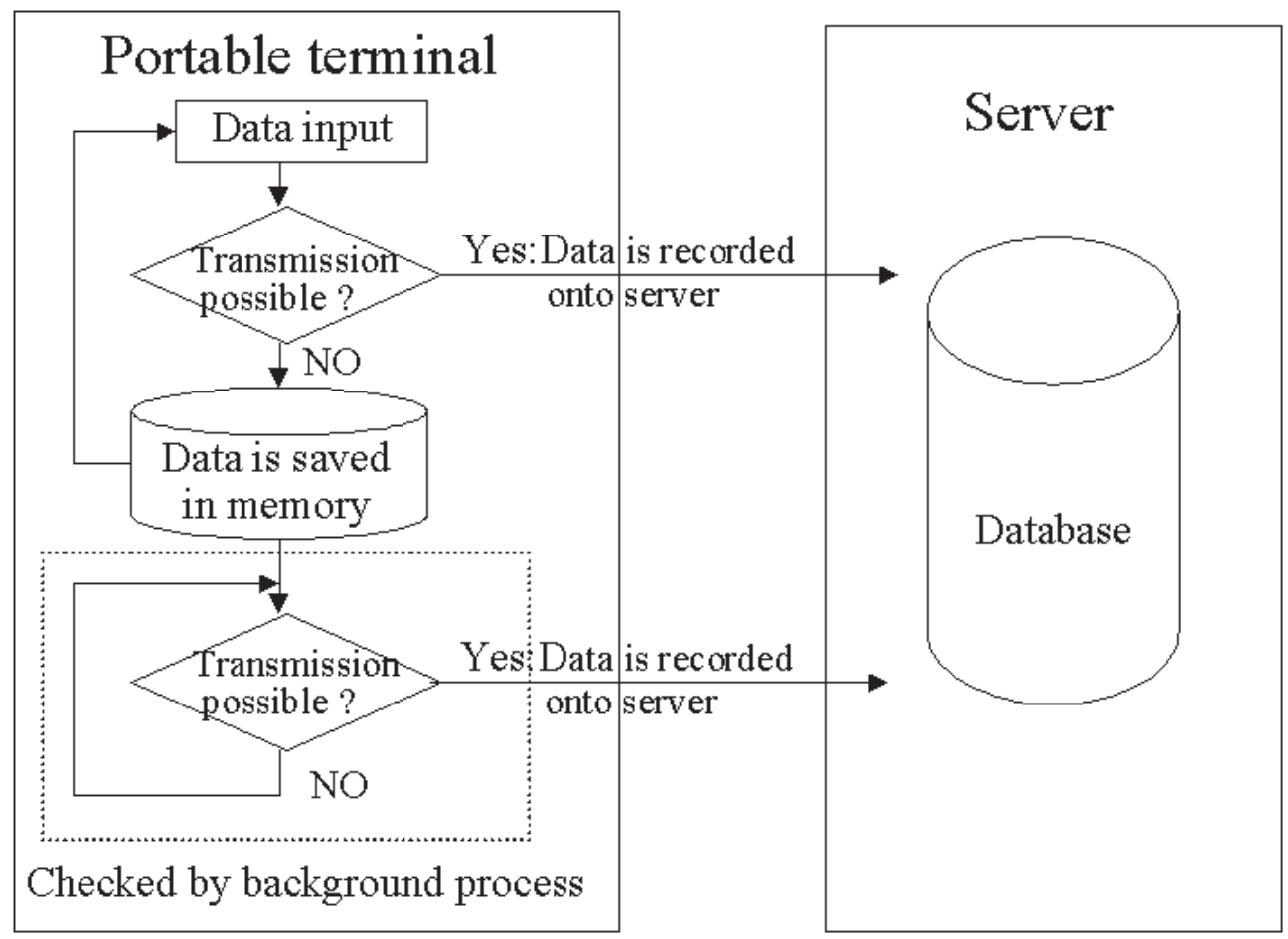

Fig. 4 Processing flow 
- When the electric wave is interrupted, data is gathered on a PDA file, and as soon as it is open, data is automatically recorded onto the database server.

We designed the data input and data transmission processes to be separate internal processes within the portable terminal, and have made the data transmission process so that it is operated as a background process. As a result, inspection data input using the PDA can be done always under similar conditions, regardless of electric wave status. Figure 4 shows the processing flow of measures to cope with electric wave interruption.

\section{Question answering system}

The form "I want to see the document (homepage) concerning AAA" is a general question demand in bibliographical databases, book search systems and Internet search engines. With such retrieval questions, it is intended that the retriever will enter the "AAA" subject expression key word and search for the document and the homepage including the expression of the character string of the "AAA."

On the other hand, the target of the question answering type search system is to retrieve "Height" and "Date" immediately on requesting "I want to know the height of Mt. Fuji" or "I want to know what date Christmas is," for instance, and it is different from the oldmodel retrieval question.

\subsection{Prototype system development}

In the railway maintenance field, the inspection maintenance manual is considered an object of the retrieval demand for a document. Each railway company prepares an original manual and standard. In these documents, the management value, the criteria, inspection items and procedures, all of which are necessary for facility maintenance, are included. However, in many cases, these manuals are kept in offices and never taken out into the field, so retrieval or reference of manual is required during actual facility inspections ${ }^{2)}$.

Therefore, we have studied a method by which the appropriate manual can be referred to easily from anywhere when necessary, and in addition, information is accessible in the form of a request, and have developed a prototype system. In this system, we used the maintenance inspection of a tunnel as an example and arranged for the type of questions to be raised regarding the maintenance manual.

\subsection{Structuring of manual document}

The manual document has a structure consisting of the chapter, the paragraph, the text, the title, and the diagram, etc. Although a person looking at it can easily understand this kind of structure of a normal text document file, it isn't an easy form for a computer to understand. Therefore, we defined the necessary structure and converted the manual document into XML form, aiming to judge an integrity degree of facility. Figure 5 shows

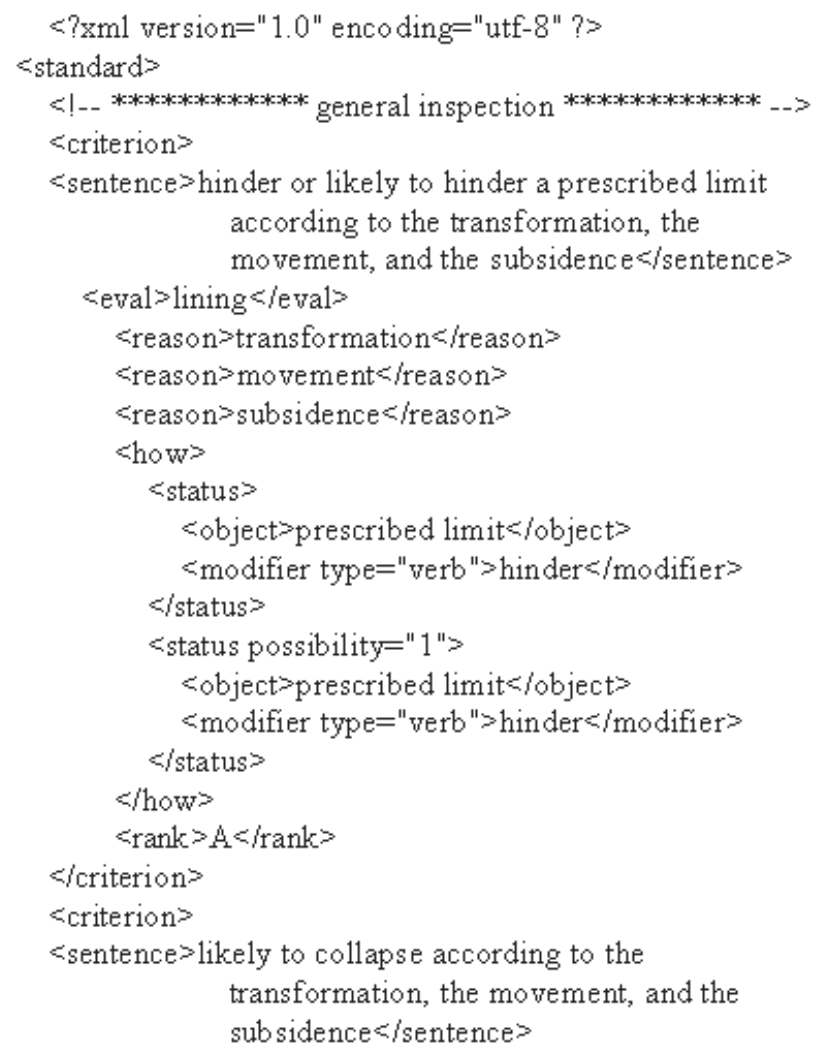

Fig. 5 Example of manual in XML form

part of the manual converted into XML form.

\subsection{Judgment processing}

When we constructed the previous question answering system, we developed an analysis-processing program for question sentences. This carried out the retrieval question analysis by the morphological analysis function and converted the retrieval question sentence into a simplified sentence by using a synonym translation table prepared beforehand with respect to the synonyms in sentences. This processing is necessary for specifying the word that is an important clue to understand the question type, synonymity and similar expressions, and for extracting the key words in sentences.

On the other hand, this time we designed the processing function so that it ranks judgment sentences according to a similarity level of the character string between the integrity degree judgment sentence and the question sentence. Concerning the similarity level judgment, we used a standard similarity level calculation method by the vector space model and made enhancements partly based on the simple evaluation result of this system ${ }^{3)}$. Now we are calculating a series of similarity levels and finding out which has the highest coincidence for only the <sentence $>$ part among the manual documents converted into XML form. Actually, it answers with the integrity degree judgment sentence that has the highest coincidence and its rank when the question to describe the facility fault is sent to the question answering system server by e-mail. Figure 6 shows an 
Question sentence: There is a water leak from the arch part

3.574 Lining in the vicinity of arch might fall.

0.778 The train operation is disturbed for the clearance disorder caused by the icicle by the water leak and freezing of the sidewall.

0.000 Because the track irregularity increases by mud-pumping of roadbed and heaving, and the period of maintenance work shortened extremely, securing normal train operation is difficult.

0.778 The state of roadbed has deteriorated by the drain obstruction and the water leak.

9.863 The water leak from the arch is transmitted directly. in the overhead line and the insulator in the electrification section.

Similarity level

The highest coincidence: 9.863

Judgment standard sentence: The water leak from the arch

is transmitted directly in the overhead line and the insulator in the electrification section.

\section{Fig. 6 Example of calculation result}

example of the result of a similarity level calculation.

\section{Evaluation and subject in the future}

a) RF-ID tag

The communication range of test system's electric wave was about $2 \mathrm{~cm}$, which is not enough for actual railway facility maintenance work. Therefore, to lengthen the communication range, it is necessary to use a tag with an internal battery or a large-scale tag with built-in big antenna and a large-scale reader.

b) Speech recognition input

The speech recognition engine of this system works independently on a PDA, and recognizes voices of multiple people, but the recognition level is not sufficient. However, it can be expected that it will reach a practical level by improving the performance of the speech recognition engine.

In addition, because one hand is used for to hold the PDA, the method for confirming the recognition result on the PDA screen is not hands-free in the strict sense of the word. Therefore, we want to examine a method using a headset mike for voice inputs with the PDA placed in the pocket, and to confirm the recognition result by the voice synthesis with the earphone.

c) Question answering system

For this, we have constructed a mechanism that finds the integrity degree judgment sentence most similar to the natural sentence question and presents the facility's integrity degree by a similarity level calculation method based on the character string coincidence level. To achieve this function, we have converted the manual document into XML form. It will be assumed to be subject to evaluate using more question examples and to compare with the morphological analysis method.

\section{Conclusion}

Portable terminals have been used for some time in facility maintenance work with the aim of improving efficiency and reducing the posting mistake. However, they have not been widely used because there have been some problems such as the size of the operation screen, the weight of the terminal itself and inadequate performance. We have introduced in this paper various experimental systems that have been developed in an attempt to overcome these problems and to add speech recognition and question answering functions according to improving portable terminal performance. We wish to advance this research to a practical stage including the examination of various aspects that remain to be resolved.

\section{References}

1) Yagi, Y., Kikuchi, M., Sato, N., and Miyashita, M., "Utilization of Mobile Internet for Facility Maintenance," RTRI Report, Vol. 15, No. 9, pp.33-36, 2001.

2) Nozue, M., Matsuoka, A., Yasuda, M., and Tanaka, M., "Development of Question Answering System by Natural Language Mail Text," RTRI Report, Vol. 17, No. 12, pp.43-48, 2003.

3) Kishida, K., Theory and technology of information retrieval. Keisosyobo, 1998 (in Japanese). 